\title{
Compact Half Diamond Dual-Band Textile HMSIW On-body Antenna
}

\author{
Sam Agneessens, Student Member, IEEE, and Hendrik Rogier, Senior Member, IEEE
}

\begin{abstract}
A novel wearable dual-band textile antenna, designed for optimal on-body performance in the $2.4 \mathrm{GHz}$ and $5.8 \mathrm{GHz}$ Industrial, Scientific and Medical bands, is proposed. By using brass eyelets and a combination of conducting and nonconductive textile materials, a Half-Mode Substrate Integrated Waveguide cavity with ground plane is realized that is very compact, flexible and, while still directing radiation away from the wearer. Additional miniaturization is achieved by adding a row of shorting vias and slots. Beside excellent free space performance in the $2.4 \mathrm{GHz}$ and $5.8 \mathrm{GHz}$ bands, respectively, with measured impedance bandwidth of $4.9 \%$ and $5.1 \%$, maximal measured free-space gain of $4.1 \mathrm{dBi}$ and $5.8 \mathrm{dBi}$, and efficiency of $72.8 \%$ and $85.6 \%$, very stable on-body performance is obtained, with minimal frequency detuning when deploying the antenna on the human body and when bent around cylinders with radii of $75 \mathrm{~mm}$ and $40 \mathrm{~mm}$. At $2.45 \mathrm{GHz}$ and $5.8 \mathrm{GHz}$, respectively, the measured on-body gain is $4.4 \mathrm{dBi}$ and $5.7 \mathrm{dBi}$, with sufficiently small calculated SAR values of $0.55 \mathrm{~W} / \mathrm{kg}$ and $0.90 \mathrm{~W} / \mathrm{kg}$. These properties make the proposed antenna excellently suited for wearable on-body systems.
\end{abstract}

Index Terms - Multifrequency antennas, Textile antennas, Half-mode substrate integrated waveguides.

\section{INTRODUCTION}

$\mathrm{B}$ ODY-WORN electronics have significant potential in various diverse applications and domains, such as positioning and tracking for firefighters; robust, low weight, integrated wireless communication devices for rescue personnel; and increasing the quality of life while decreasing the cost for the elderly, the disabled or medical patients by providing integrated remote medical monitoring, amongst others [1], [2]. These systems have received a lot of attention and many studies, in a broad application range, are found in literature, such as (but not limited to): integrated radar for firefighters [3], search and rescue systems in lifejackets [4], and wearable biosensor systems [5].

In achieving such high-performance, unobtrusive, wearable systems, a high level of integration and a dedicated antenna design formalism are vital. As such, textile antennas provide a popular solution as their flexibility and low weight enables them to excellently cope with the dynamic environment of the human body (moving, twisting, turning), while maintaining

S. Agneessens and H. Rogier are with the Department of Information Technology (INTEC), Ghent University, St. Pietersnieuwstraat 41, 9000 Ghent, Belgium (E-mails: Sam.Agneessens@UGent.be, Hendrik.Rogier@UGent.be). the wearer's comfort. In addition, they can achieve a performance comparable to that of antennas on rigid substrates.

The 2.4 GHz Industrial Scientific and Medical (ISM) band has proven to be very well suited for these smart e-textile systems and several studies presented textile antennas operating in this unlicensed band [6],[7], [8]. Other designs have received their fair share of attention, such as antennas for dual and multiband operation [9], [10],[11], [12], [13], designs with UWB performance [14] or large fractional bandwidth to cover multiple applications, and topologies that apply Electromagnetic Band Gap (EBG) structures in the textile substrate to optimize performance near the human body [15], [10].

Most of these textile antenna designs are planar topologies based on microstrip or CoPlanar Waveguide (CPW) patches. They are low profile and robust, but require a ground plane or EBG substrate with dimensions larger than those of the patch to adequately shield the body of the human wearer from the radiating antenna. Designs that do not use such a technique suffer from large degradation of radiation performance and high Specific Absorption Rate (SAR) values when placed in too close proximity of the body [16], [17]. They require thick textile layers to provide sufficient spacing before they can be used in a practical application.

Recently, a new textile antenna topology was presented [18]: a $2.4 \mathrm{GHz}$ ISM band cavity backed textile antenna applying Substrate Integrated Waveguide (SIW) technology, which provides increased isolation between antenna and wearer without the need for a large ground plane.

SIW technology leverages a low-cost, low-loss implementation of planar microwave components and offers a performance comparable to that of a rectangular waveguide while being compact and compatible with printed circuit board technology. SIW antennas come in many forms and operational principles. Interesting structures for on-body use range from cavity backed slot antennas [19], [20], over leaky waveguides [21] to Half-Mode [22] and Quarter Mode leaky cavities [23].

In this paper, a new Half-Mode Substrate Integrated Waveguide (HMSIW) topology is implemented in textile materials to achieve a novel, compact and easy-to-fabricate structure, very suitable for dual-band on-body operation thanks to its combination of small dimensions, low SAR values and very robust on-body performance. 


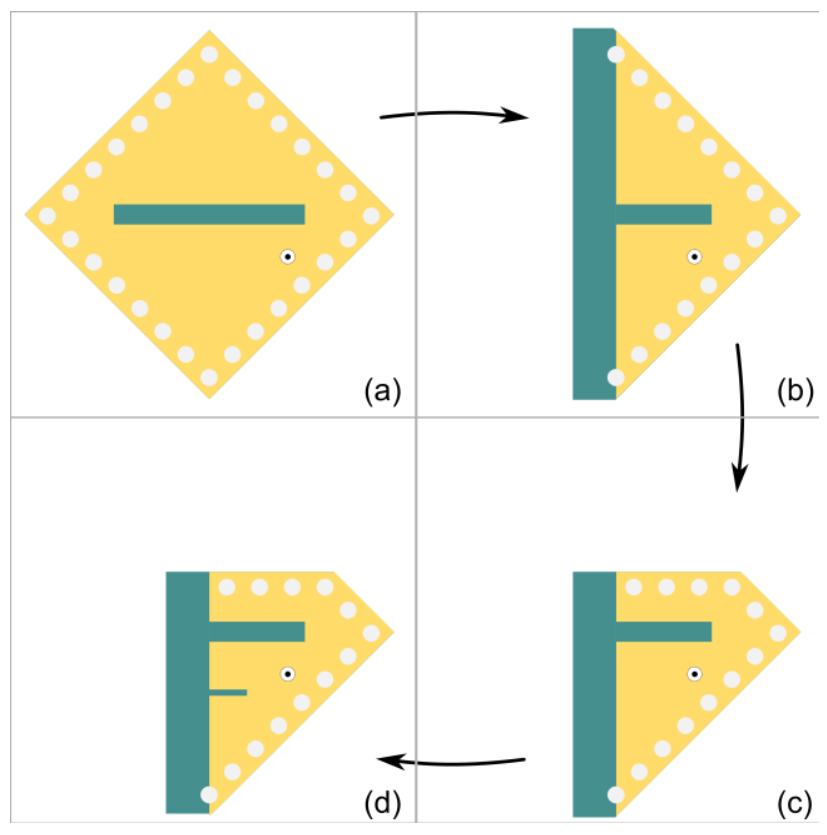

Fig. 1. Miniaturization strategy (a) starting point: cavity backed slot antenna, (b) use virtual magnetic wall to reduce dimensions, (c) further size reduction by adding shorting pins, (d) additional slot to increase bandwidth in higher frequency range and fine tune the resonance frequencies.

Specifically, the antenna exhibits a HMSIW cavity backed slot topology, with a diamond shape, leveraging a ground plane and SIW technology to increase the isolation between the human body and the radiating element. In contrast to the HMSIW textile antenna presented in [24], this design features dual-band operation, functioning both in the $2.4 \mathrm{GHz}$ ISM band, extending from $2.4 \mathrm{GHz}$ to $2.4835 \mathrm{GHz}$, as well as in 5.8 $\mathrm{GHz}$ ISM band, ranging from $5.725 \mathrm{GHz}$ to $5.875 \mathrm{GHz}$, making it suitable for the Zigbee, IEEE 802.11n (Wi-Fi), wireless HDMI and Bluetooth wireless standards. The use of a ground plane makes the antenna much simpler, smaller and easier to fabricate than designs using EBG substrates [10] to separate body and antenna, while maintaining excellent on-body robustness, radiation performance and low SAR values.

This novel HMSIW textile design yields a significant reduction in size in comparison to conventional textile patch antennas [6],[7], [8], which require an extended ground plane to sufficiently shield the human body. Its dimensions are also significantly smaller than other dual-band textile designs, being $79.2 \%$ smaller than the suspend-plate design presented in [13], and yielding a $85.5 \%$ size reduction with respect to the dual-band wearable antenna on an EBG substrate [10]. Compared to other compact dual-band textile antennas [11], [25], the proposed design has a very robust on-body performance, with low frequency detuning and radiation performance deterioration when placed on-body and under bending conditions. With respect to other on-body SIW designs, such as [18] and [24], the presented design adds dualband functionality and achieves additional size reduction by relying on a shorting wall of vias. Furthermore, this study presents the first experimental investigation of the

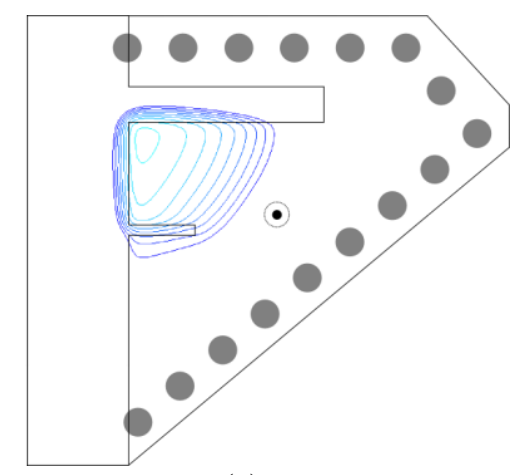

(a)

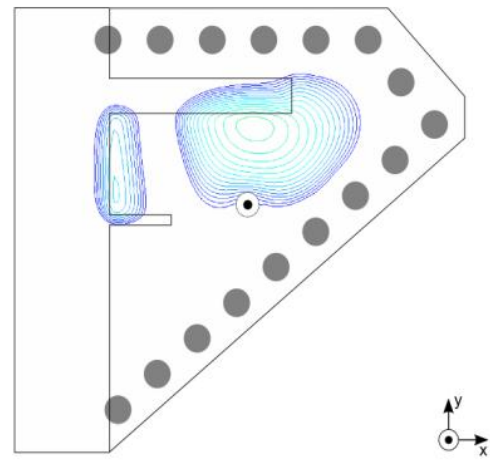

(b)

Fig. 2. E-field distribution in the HMSIW cavity at (a) $2.45 \mathrm{GHz}$ and (b) $5.8 \mathrm{GHz}$

performance of an HMSIW antenna in an on-body environment.

Section II of the paper discusses the design and fabrication of the novel on-body antenna, which is tested, measured and validated in free space and in the body environment with results discussed in Section III. Section IV covers the conclusions and outlook.

\section{HALF-DIAMOND HMSIW ANTENNA}

\section{A. Antenna design specifications}

A small size, high performance, on-body antenna is designed, operating in the 2.4 and $5.8 \mathrm{GHz}$ ISM bands. Therefore, an impedance matching criterion $\left|S_{11}\right|<-10 d B$, with respect to $50 \Omega$, in the frequency bands $2.4-2.5 \mathrm{GHz}$ and $5.725-5.875 \mathrm{GHz}$, and a radiation efficiency better than $50 \%$ are imposed.

For on-body use, it is vital that the antenna is low-weight, flexible and has low overall dimensions, while keeping in mind the SAR guidelines for EM radiation absorption (maximal absorbed power $1.6 \mathrm{~W} / \mathrm{kg}$ over an averaged region of $1 \mathrm{~g}$ tissue) [26]. The influence of factors such as movement, variations in body morphology and position of the antenna on the body should be minimized to achieve stable radiation performance and a stable reflection coefficient (i.e. a robust antenna) under very different conditions.

\section{B. Antenna topology}

To meet these stringent on-body requirements, a-to the 


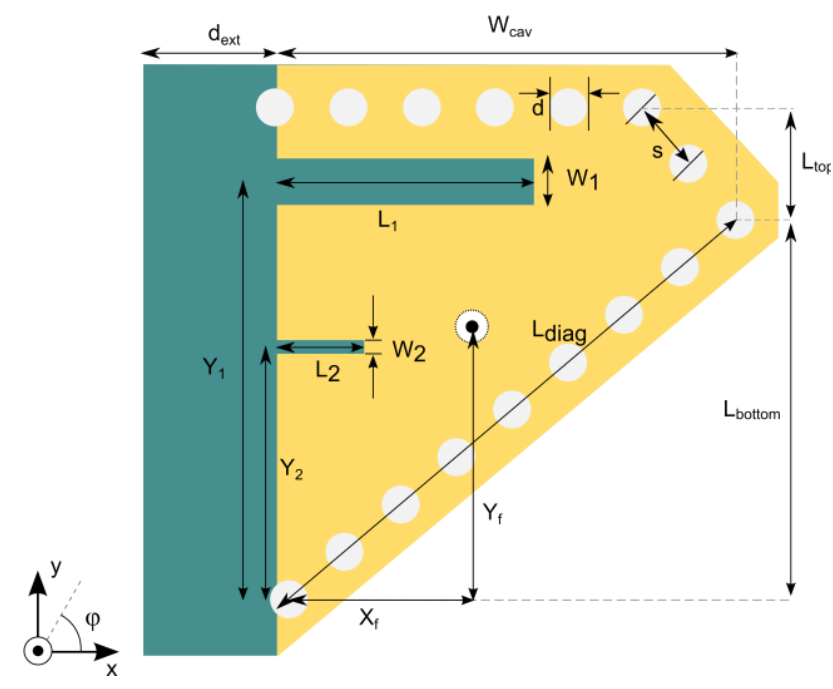

Fig. 3. Layout of the proposed HMSIW dual-band textile antenna.

best of the authors' knowledge - novel antenna topology is proposed: a half diamond shaped cavity backed slot dual band HMSIW antenna. Fig. 1 demonstrates how a single frequency SIW cavity backed slot antenna with the slot along the diagonal of the square cavity is transformed into this innovative topology.

Given the symmetry of the electric field distribution in the cavity, the symmetry plane behaves as a virtual magnetic wall. Therefore, following Fig.1.b, the cavity is split along this plane, leading to a size reduction of almost $50 \%$ without significant loss in radiation performance and impedance matching.

This type of HMSIW cavity backed slot antenna can be circularly polarized, as in [22], where two different modes with a $90^{\circ}$ phase shift are excited in the cavity, one in the upper and one in the lower half of the half mode cavity separated by the slot. Yet, in this design an additional size reduction is achieved by dropping the circular polarization requirement. Then, only the mode in the lower half of the cavity (the region below the largest slot in Fig. 2) needs to be excited to give rise to radiation and the area in the upper half (the region above the largest slot in Fig. 2) can be shorted, leading to an even smaller antenna, as shown in Fig. 1.c. By optimizing the position of the feed point, a second-order mode at a higher frequency is excited, making the structure suitable for dual band operation.

Finally, the resonance frequency in the higher frequency band is tuned by adding a second slot, as shown in Fig. 1.d. Enlarging this additional slot decreases the resonance frequency of the second-order mode and provides larger bandwidth in the higher frequency band.

\section{Computer-aided design process}

The design strategy for a half diamond HMSIW antenna fabricated from textile materials is outlined, keeping in mind the design requirements in Section II.A. As antenna substrate a flexible closed-cell expanded rubber protective foam with thickness $\mathrm{h}=3.94 \mathrm{~mm}, \varepsilon_{\mathrm{r}}=1.495$ and $\tan \delta=0.016$ is chosen.

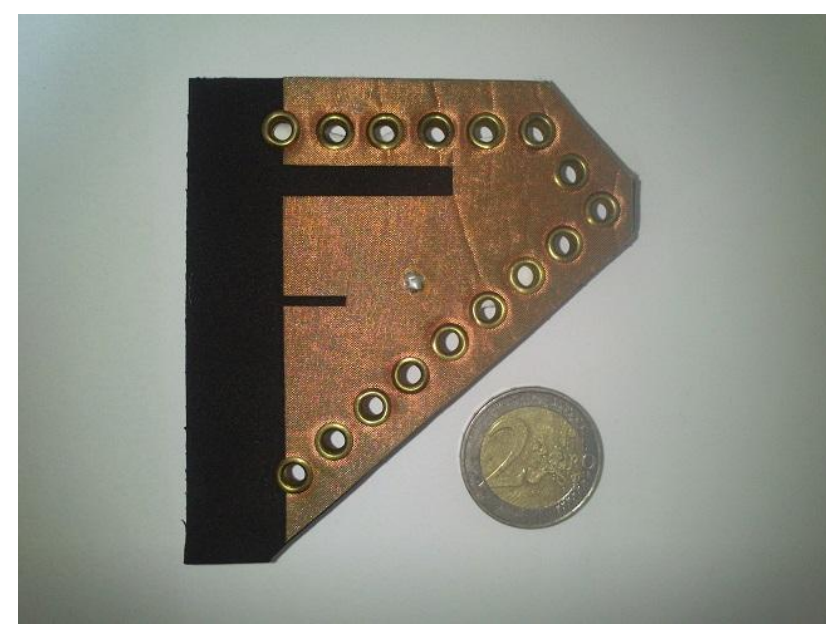

Fig. 4. Photograph of the fabricated textile dual-band HMSIW on-body Antenna.

The dielectric parameters of the textile materials are extracted by means of the matrix-pencil two-line method [27]. The conductive planes are fabricated from a copper plated polyester taffeta fabric with thickness $80 \mu \mathrm{m}$ and surface resistivity $0.18 \Omega / \mathrm{sq}$ at $2.45 \mathrm{GHz}$. The antenna geometry is cut by hand from the textile material by using a scalpel. To realize the vias in the design, brass eyelets are used. Concerning via

TABLE I

PROPOSED HMSIW DUAL BAND TEXTILE ANTENNA DIMENSIONS

\begin{tabular}{clrl}
\hline Parameter & Value & Parameter & Value \\
\hline $\mathrm{W}_{\text {cav }}$ & $50.0 \mathrm{~mm}$ & $\mathrm{~d}$ & $4 \mathrm{~mm}$ \\
$\mathrm{~L}_{1}$ & $28.0 \mathrm{~mm}$ & $\mathrm{~s}$ & $8 \mathrm{~mm}$ \\
$\mathrm{~W}_{1}$ & $4.9 \mathrm{~mm}$ & $\mathrm{X}_{\mathrm{f}}$ & $20.0 \mathrm{~mm}$ \\
$\mathrm{~L}_{2}$ & $9.4 \mathrm{~mm}$ & $\mathrm{Y}_{\mathrm{f}}$ & $29.8 \mathrm{~mm}$ \\
$\mathrm{~W}_{2}$ & $1.5 \mathrm{~mm}$ & $\mathrm{~L}_{\text {top }}$ & $12.3 \mathrm{~mm}$ \\
$\mathrm{Y}_{1}$ & $45.7 \mathrm{~mm}$ & $\mathrm{~L}_{\text {bottom }}$ & $41.4 \mathrm{~mm}$ \\
$\mathrm{Y}_{2}$ & $27.5 \mathrm{~mm}$ & $\mathrm{~L}_{\text {diag }}$ & $65.6 \mathrm{~mm}$ \\
$\mathrm{~d}_{\text {ext }}$ & $14.6 \mathrm{~mm}$ & & \\
\hline
\end{tabular}

spacing, the SIW guidelines are taken into account: $s \leq 2 d$ and $d / \lambda_{f, \max } \leq 0.1$ [19]. They are put in place with a hand press, which enables a more precise placement of the vias than in [18].

The antenna is resonance based; two resonating modes are excited in the cavity which radiate into space through the open side wall and - to a lesser extent - through the slots. The Efield distribution at the two center frequencies $(2.4 \mathrm{GHz}$ and $5.8 \mathrm{GHz}$ ) is shown in Fig. 2.

Fig. 2. also helps clarify the antenna's polarization at the different frequencies of operation. At both center frequencies there is a contribution to the far-field E-field which originates from the open wall of the cavity between the two slots. At $2.45 \mathrm{GHz}$ the other main contributor is the radiation from the largest slot. The combination of these two components leads to an E-field which is polarized along $\varphi=135^{\circ}$. For the higher frequency band both slots radiate, but the magnitude of radiation from the smaller slot is much larger than that of the 


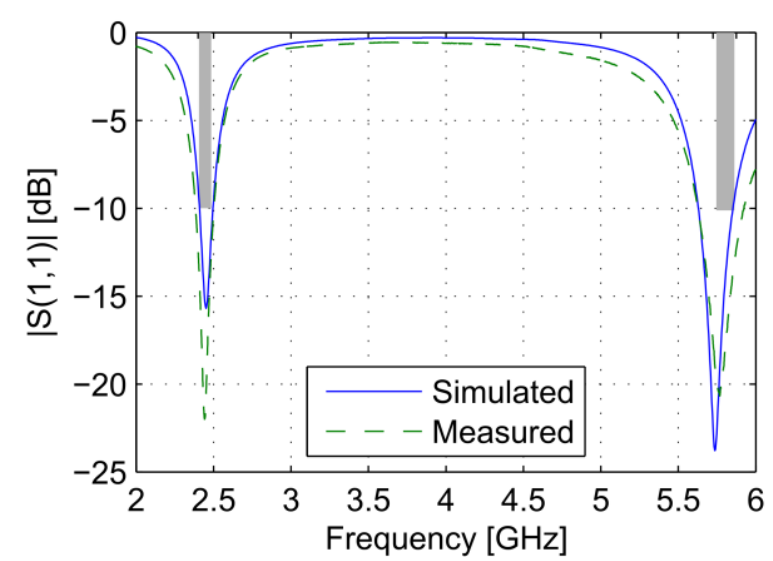

Fig. 5. Simulated and measured free space reflection coefficient

longer slot, causing radiation from the small slot to be the second the dominant contributor, besides the radiation from the open wall. This leads to linear polarization along $\varphi=45^{\circ}$ at $5.8 \mathrm{GHz}$.

Therefore, the first step in the design process consists of determining the dimensions of the cavity to fix the frequency of the resonant modes. The starting point is a half open diamond cavity with two slots in the top plane, such as shown in Fig. 3, with the diagonal length of the cavity, $L_{\text {diag }}$, equal to half a wavelength and the length of the large slot, $\mathrm{L}_{1}$, to a quarter wavelength at the lowest desired resonance frequency, with the slot positioned at the broadest point of the cavity. The short slot length also corresponds to a quarter wavelength, but at the center frequency of the higher ISM band. It is positioned below the long slot, at a distance $Y_{1}-Y_{2}=$ $f_{\text {low }} / f_{\text {high }} \cdot \lambda_{\text {low }} /\left(2 \sqrt{\varepsilon_{r}}\right)$. This rule-of-thumb is based on a large set of numerical simulations and parameter sweeps.

A coaxial feed, placed near the cavity wall, between the two slots, is used with the outer conductor connected to the bottom plane and the inner connector to the top plane, creating a loop to inject energy into the cavity by coupling to the magnetic fields of the resonating modes. The position of the feed, defined by $X_{\mathrm{f}}$ and $\mathrm{Y}_{\mathrm{f}}$, is important to achieve good matching. The probe feed should be close enough to the diagonal via side wall, to achieve good coupling to the magnetic field of the modes, and should not be too close to the maxima of the E-fields of the modes to be excited.

A smaller antenna size can be achieved by reducing $d_{\text {ext }}$, but the increased compactness comes at the price of decreased on-body antenna efficiency. The reduction of $d_{\text {ext }}$ results in larger back radiation and, hence, decreased radiation efficiency when the antenna is worn by a person. Therefore, it is important to choose a value for $d_{\text {ext }}$ that yields an optimal balance between on-body radiation efficiency and compactness.

Parameters that change the shape of the cavity confining the resonant modes - such as $\mathrm{L}_{\text {top }}$, position and length of the slots, as well as $\mathrm{W}_{\text {cav }}$ - have a distinct influence on the frequency response and are important in fine tuning the antenna performance.
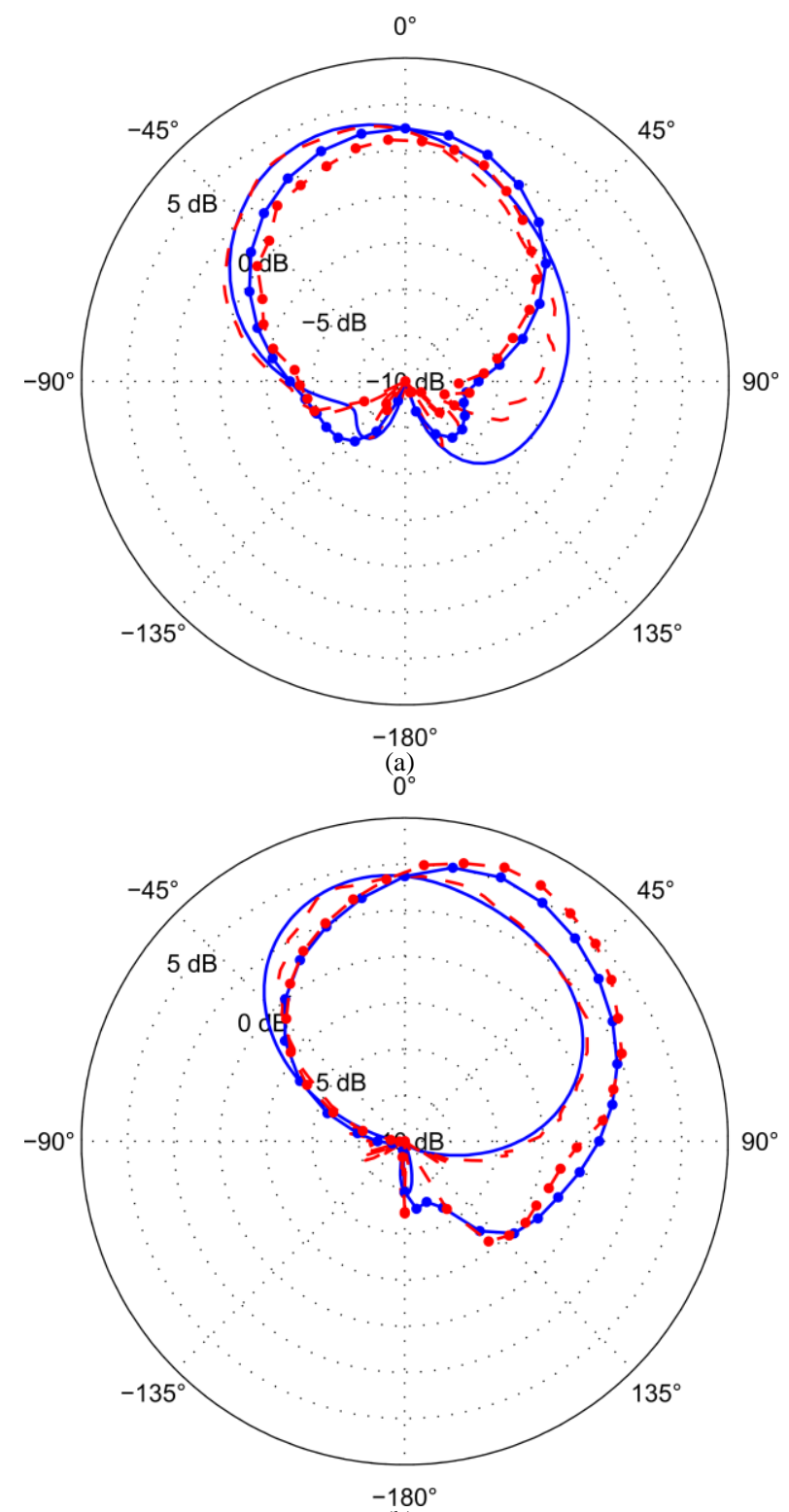

(b)

Fig. 6. Simulated (full line) and measured (dashed line) free space total gain in the E-plane (no markers) and H-plane (dot markers) at (a) $2.4 \mathrm{GHz}$ (cut along $\varphi=135^{\circ}$ ) and (b) $5.8 \mathrm{GHz}$ (cut along $\varphi=45^{\circ}$ ).

$\mathrm{L}_{\text {top }}$ can be used to tune the higher resonance frequency independently from the lower resonance frequency. Decreasing $\mathrm{L}_{\text {top }}$ reduces the cavity size relevant to the second mode (see Fig. 2.), thereby increasing the related resonance frequency.

The offset of the slots along the y-axis also changes the structure's dimensions and hence the possible mode profiles. Decreasing the values of $Y_{1}$ and $Y_{2}$ equally will shift the resonance frequencies upward, because the resonating modes will be confined in a smaller section of the triangular cavity. Moving both slots simultaneously is useful when it is necessary to tune the frequency response of the design in both bands at the same time. It is important to keep in mind that for 

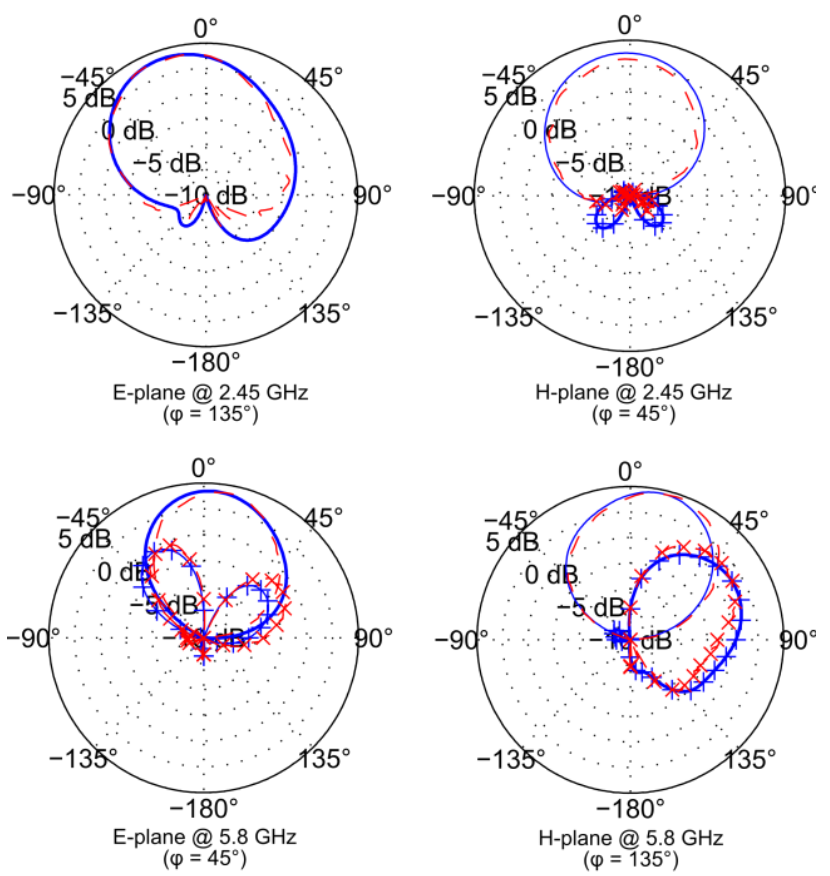

$\left(\varphi=135^{\circ}\right)$

Fig. 7. Simulated (full line) and measured (dashed line) free space co- (no marker) and cross-polarization (simulation: +-marker, measurement: $\mathrm{x}$-marker) for the $\mathrm{E}$ - and $\mathrm{H}$-plane at $2.45 \mathrm{GHz}$ and 5.8 $\mathrm{GHz}$.

this simultaneous tuning, the position of the feed should be kept constant relative to the positions of both slots.

Similar to the offset of the slots, the slots' lengths also define the area confining the modes. An increase in length leads to a downward shift in resonance frequency.

A larger cavity width, $\mathrm{W}_{\text {cav }}$, results in a downward shift for both modes due to the larger cavity size. Similar to the slots' offset, the distance to the via sidewall of the feed should remain constant when sweeping this parameter.

By tuning these parameters, an antenna geometry was obtained with two resonating modes, close to the desired frequency bands. Further optimization of the dimensions, to fulfill the specifications, is performed with the full-wave 3D electromagnetic simulation tool CST Microwave Studio ${ }^{\circledR}$ [28]. Details about the final design's geometry are presented in Table I and Fig. 3. A photograph of the final fabricated antenna is shown in Fig. 4. The HMSIW antenna has a total surface area of $2080 \mathrm{~mm}^{2}$ which is $85.5 \%$ smaller than the design presented by [10].

\section{MeAsurement Results}

A half diamond HMSIW antenna prototype was fabricated from textile materials and tested under free space conditions to validate the design and its performance. The results are discussed in section III.A.

Since the human body is a very specific environment for an antenna, the antenna's behavior can vary significantly from that in free space. If the antenna is not designed carefully the body environment can significantly reduce its performance: impedance matching deterioration, frequency detuning and tremendous radiation performance degradation. In section

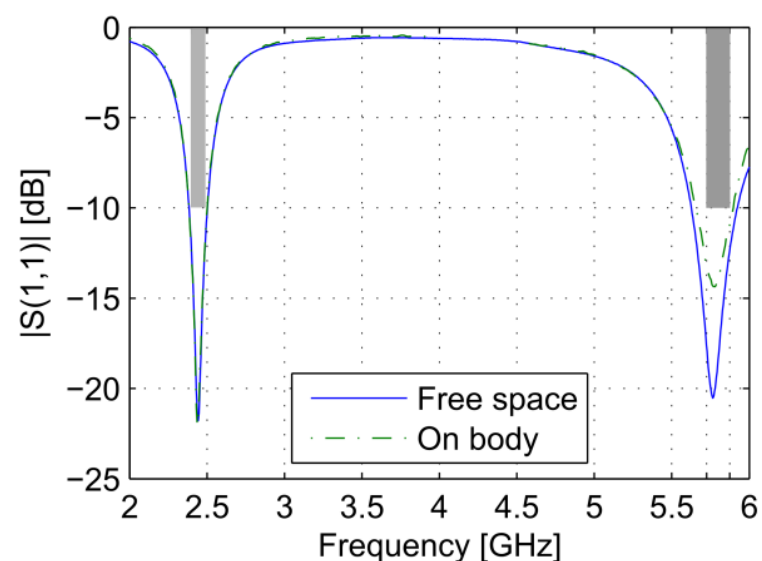

Fig. 8. Measured on-body reflection coefficient versus free space measurement.

III.B, measurements and experiments are presented to verify the performance when bending the antenna and placing it in proximity of the human body.

\section{A. Figures of merit in free space}

The network parameters and radiation performance of the proposed design in free space are measured using an Agilent N5242A PNA-X Microwave Network Analyzer [29], with the antenna placed in an anechoic chamber. The reflection coefficients of the simulated and measured antenna are plotted in Fig. 5. Good agreement between the simulated and measured values is observed and the antenna completely covers the $2.4 \mathrm{GHz}$ ISM band $(2.4 \mathrm{GHz}-2.4835 \mathrm{GHz})$ and $5.8 \mathrm{GHz}$ ISM band $(5.725 \mathrm{GHz}-5.875 \mathrm{GHz})$, with a measured fractional bandwidth of $4.9 \%$ and $5.1 \%$, respectively.

The radiation pattern and radiation efficiencies at the center frequency of the two frequency bands were validated by 3D measurements in the anechoic chamber. The measured and simulated free space gains in the E-plane $\left(\varphi=135^{\circ}\right.$ at 2.45 $\mathrm{GHz}$ and $\varphi=45^{\circ}$ at $\left.5.8 \mathrm{GHz}\right)$ and H-plane $\left(\varphi=45^{\circ}\right.$ at $2.45 \mathrm{GHz}$ and $\varphi=135^{\circ}$ at $5.8 \mathrm{GHz}$ ) are shown at $2.45 \mathrm{GHz}$ in Fig 6.a and at $5.8 \mathrm{GHz}$ in Fig. 6.b. Again, there is good agreement between the measured and the simulated radiation patterns. At $2.45 \mathrm{GHz}$, the maximum simulated and measured gains are $4.2 \mathrm{dBi}$ and $4.1 \mathrm{dBi}$, respectively, and at $5.8 \mathrm{GHz}$ the maximum simulated and measured values are $5.5 \mathrm{dBi}$ and $5.8 \mathrm{dBi}$. The measured radiation efficiency at $2.45 \mathrm{GHz}$ amounts to $72.8 \%$ and $85.6 \%$ at $5.8 \mathrm{GHz}$, which proves that the structure operates as an efficient antenna at these frequencies.

The co- and cross-polarization for the E- and H-plane at both $2.45 \mathrm{GHz}$ and $5.8 \mathrm{GHz}$ are shown in Fig. 7. At $2.45 \mathrm{GHz}$, the antenna is strictly linearly polarized along the axis $\varphi=$ $135^{\circ}$. The cross-polarization for the E-plane remains lower than $-10 \mathrm{dBi}$ for all angles. At $5.8 \mathrm{GHz}$, the antenna is linearly polarized in broadside direction, but for large elevation angles there is a significant cross-polarized component.

\section{B. On-Body Performance}




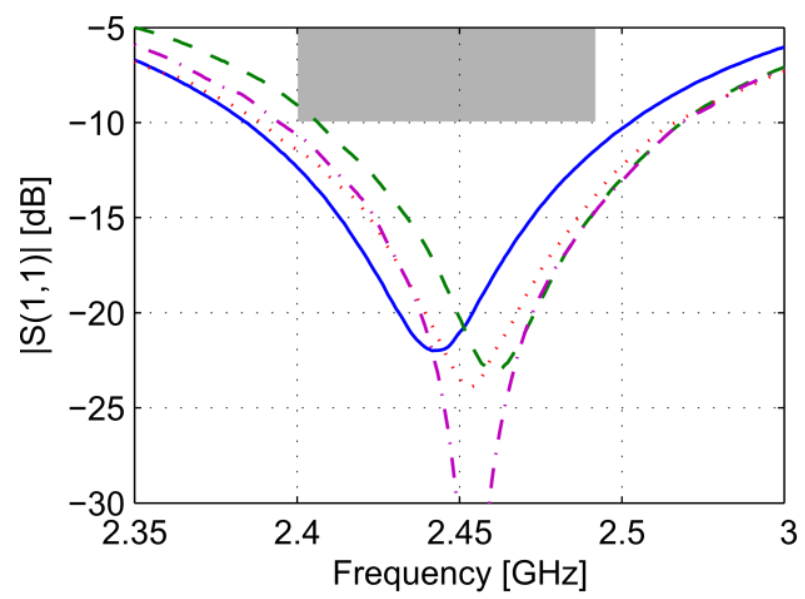

(a)

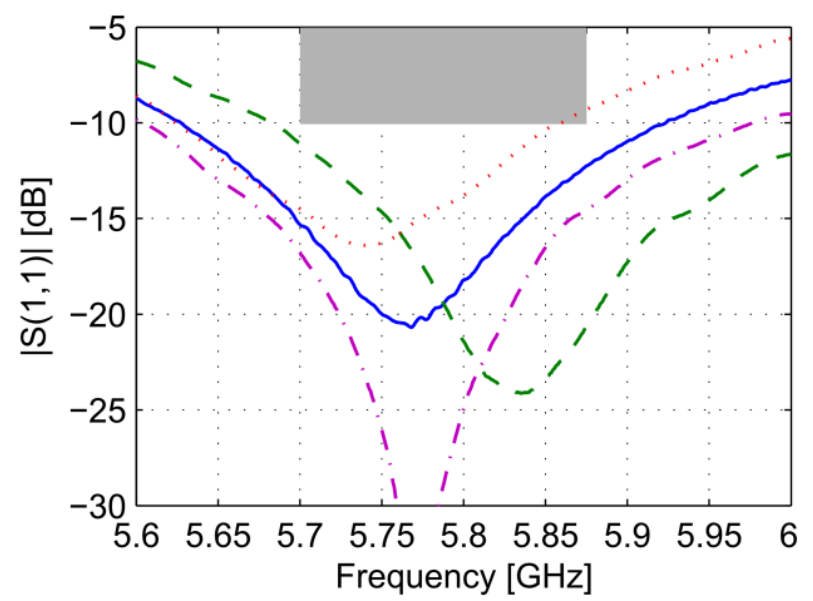

(b)

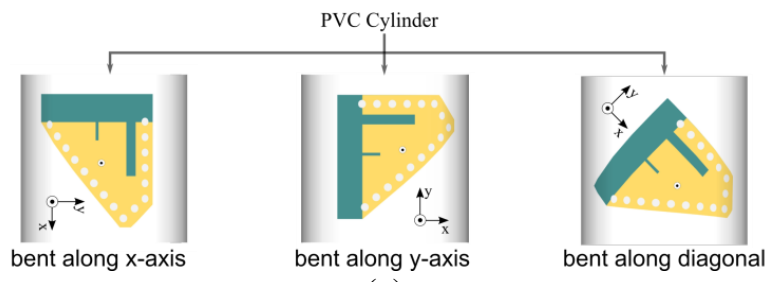

(c)

Fig. 9. Measured reflection coefficient during bending considering the (a) $2.4 \mathrm{GHz}$ ISM band and (b) $5.8 \mathrm{GHz}$ ISM band. The antenna is bent along the $\mathrm{x}$-axis (dashed line), the $\varphi=45^{\circ}$ diagonal (dotted line) and the $y$-axis (dash-dotted line) for radius: $40 \mathrm{~mm}$. (c) The bending measurement setup.

Fig. 8 shows the measured reflection coefficient of the antenna placed on the body, with a $1 \mathrm{~mm}$ thick textile layer in between body and antenna, compared to the free space situation. Very stable on-body performance is observed: the matching around $2.45 \mathrm{GHz}$ remains unchanged and, in the $5.8 \mathrm{GHz}$ band, the bandwidth is slightly reduced, but the reflection coefficient remains well below $-10 \mathrm{~dB}$ in the entire band.

The influence of bending was verified by wrapping the antenna prototype around two polyvinyl chloride (PVC) cylinders with different radii: $40 \mathrm{~mm}$ and $75 \mathrm{~mm}$. These two configurations mimic bending of the wearable antenna when deployed on a very thin arm or leg, respectively. As depicted

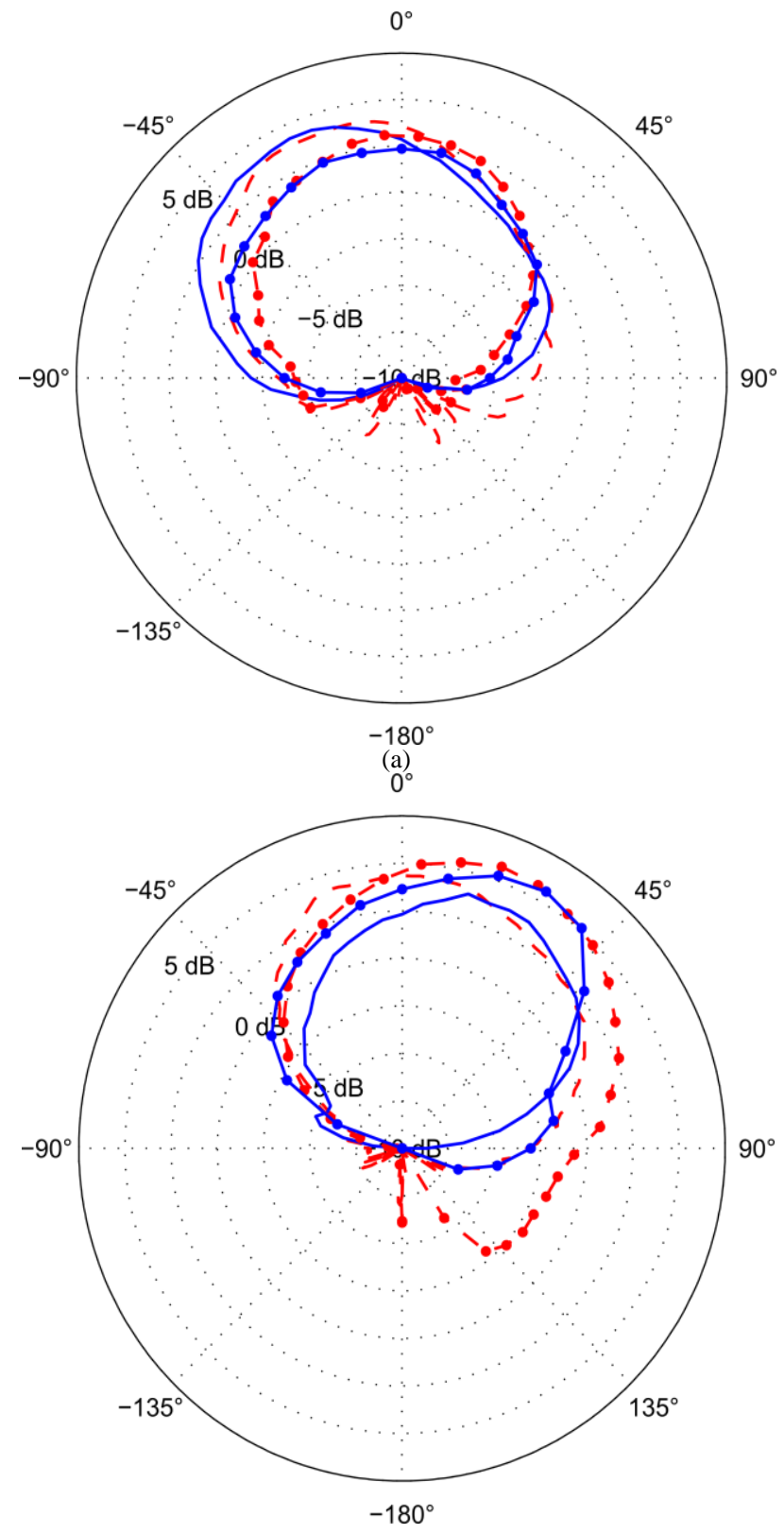

(b)

Fig. 10. Measured free-space gain (dashed line) and on-body gain (full line) in the E-plane (no marker) and H-plane (dot marker) at (a) $2.45 \mathrm{GHz}$ and (b) $5.8 \mathrm{GHz}$.

in Fig. 9.c, three bending directions are considered: along the $\mathrm{x}$-axis, along the $\mathrm{y}$-axis and along the diagonal axis $\left(\varphi=45^{\circ}\right.$ in Fig. 3). The measured reflection coefficients, for the smallest bending radius of $40 \mathrm{~mm}$, are displayed in Fig. 9. In the lower frequency band, the impedance matching criterion of $\left|S_{11}\right|<$ $-10 d B$ remains satisfied when bending along the y-axis and the $\varphi=45^{\circ}$ diagonal. When bending along the $x$-axis, the matching requirement is not met at $2.4 \mathrm{GHz}$, with a maximum reflection coefficient under these extreme bending conditions of $-9.1 \mathrm{~dB}$. Yet, this value still ensures good antenna operation. Also in the higher frequency band, the complete 5.8 $\mathrm{GHz}$ ISM band remains covered under all but one of the tested conditions. For the extreme bending radius of $40 \mathrm{~mm}$ along 
the $\varphi=45^{\circ}$ diagonal, the matching criterion is not met at the $5.875 \mathrm{GHz}$ limit, with a maximum $\left|S_{11}\right|$ value of $-9.3 \mathrm{~dB}$. The measurements with the antenna bent around the cylinder with the larger radius $75 \mathrm{~mm}$ show no violations of the matching criteria in both ISM bands. Therefore, it is concluded that the antenna performance remains robust under bending as only very small out-of-band detuning occurs under the small bending radii tested.

On-body radiation performance is verified by placing the antenna on the chest of a human test subject, with a $1 \mathrm{~mm}$ layer of textile in between, and measuring the radiation pattern in the anechoic room. The design is placed on the stomach of the test subject with a body mass index of 21.5. Due to the limitations of the measurement setup - it is not possible to perform a $3 \mathrm{D}$ measurement with a person - the antenna was reoriented to be able to measure the field pattern in the E- and H-planes. Fig. 10 shows the measured radiation pattern in the E- and H-plane for both center frequencies when the antenna is placed on the human body. A maximum measured on-body gain of $4.4 \mathrm{dBi}$ is obtained at $2.45 \mathrm{GHz}$ and $5.7 \mathrm{dBi}$ at $5.8 \mathrm{GHz}$. The pattern in the E- and H-planes at $2.45 \mathrm{GHz}$ and $5.8 \mathrm{GHz}$ is slightly deformed when the antenna is placed on the body, but the shape and absolute values of the measured gain still closely resemble the measured free space performance. When deploying the antenna on the human body, the simulated radiation efficiency amounts to $61 \%$ at $2.45 \mathrm{GHz}$ and $69 \%$ at $5.8 \mathrm{GHz}$. It can be concluded that the proposed antenna is well-suited for on-body use. By increasing the extension of the ground plane, $\mathrm{d}_{\mathrm{ext}}$, the isolation between the body and the antenna improves. This will reduce unwanted body-related performance changes, such as the larger radiation pattern deformation at $5.8 \mathrm{GHz}$, caused by the body acting as a better reflector at higher frequencies, and the higher losses at $2.45 \mathrm{GHz}$, resulting from the deeper penetration of the EM-field into the human tissue.

To determine the absorbed radiation by the human tissue, the Specific Absorption Rate (SAR) values are calculated using CST Microwave Studio and the three layer human body model [30] where the antenna is placed at $2 \mathrm{~mm}$ distance from the human tissue. The calculated maximum SAR values for $500 \mathrm{~mW}$ input power at $2.45 \mathrm{GHz}$ and $5.8 \mathrm{GHz}$ are $0.55 \mathrm{~W} / \mathrm{kg}$ and $0.90 \mathrm{~W} / \mathrm{kg}$ averaged over $1 \mathrm{~g}$ of tissue. These values are well below the maximal limit of $1.6 \mathrm{~W} / \mathrm{kg}$ averaged over $1 \mathrm{~g}$ of tissue, a result which could be expected, as the good agreement between measured free-space and on-body radiation pattern and the stable reflection coefficient already indicated that most of the radiation is directed away from the body thanks to the use of a ground plane and vias.

\section{CONCLUSIONS}

A dual-band wearable antenna based on a novel half-diamond shaped HMSIW topology, fabricated using textile materials and brass eyelets, was proposed. The design was specifically conceived to exhibit robust behavior when worn by a person, by relying on a combination of a ground plane and SIW technology to create a half-open cavity.

The antenna was thoroughly tested in free-space and on-body scenarios in an anechoic chamber. Very good agreement between simulations and measurements in terms of impedance matching and radiation performance in free space is observed. Both the $2.4 \mathrm{GHz}$ and $5.8 \mathrm{GHz}$ ISM bands are covered, with a measured bandwidth of $4.9 \%$ and $5.1 \%$, maximal measured gains of $4.1 \mathrm{dBi}$ and $5.8 \mathrm{dBi}$, and measured radiation efficiency of $72.8 \%$ and $85.6 \%$, respectively.

To validate the design as a suitable on-body antenna, measurements were conducted when the antenna was bent along different axes and when placed on the human body. The impedance matching proved to be very stable even when bent around a cylinder with a very small radius of $40 \mathrm{~mm}$. Very low frequency detuning and stable radiation patterns are seen when the antenna was placed on-body, with a measured maximal gain of $4.4 \mathrm{dBi}$ and $5.7 \mathrm{dBi}$, at $2.45 \mathrm{GHz}$ and $5.8 \mathrm{GHz}$, respectively. Finally the SAR values were calculated for both frequency bands. The maximal SAR values of $0.55 \mathrm{~W} / \mathrm{kg}$ and $0.90 \mathrm{~W} / \mathrm{kg}$ for $500 \mathrm{~mW}$ input power in the $2.4 \mathrm{GHz}$ and $5.8 \mathrm{GHz}$ band are well below the limit of $1.6 \mathrm{~W} / \mathrm{kg}$ averaged over $1 \mathrm{~g}$ of tissue.

These simulations and measurements demonstrated that the antenna provides excellent on-body performance. This in addition to its low-weight, compact dimensions and dual-band operation, makes the antenna very well suited for body-worn applications: for example, as a component in systems for wearable biosensors and medical monitoring, or systems aimed at assisting rescue and security personnel.

\section{ACKNOWLEDGMENT}

Part of this work was supported by BELSPO through the IAP Phase VII BESTCOM project.

\section{REFERENCES}

[1] S. Park and S. Jayaraman, "Enhancing the quality of life through wearable technology," Engineering in Medicine and Biology Magazine, IEEE, vol. 22, no. 3, pp. 41-48, 2003.

[2] F. Axisa, P. Schmitt, C. Gehin, G. Delhomme, E. McAdams and A. Dittmar, "Flexible technologies and smart clothing for citizen medicine, home healthcare, and disease prevention," Information Technology in Biomedicine, IEEE Transactions on, vol. 9, no. 3, pp. 325-336, 2005.

[3] S. Agneessens, P. V. Torre, F. Declercq, B. Spinnewyn, G. J. Stockman, H. Rogier and D. Vande Ginste, "Design of a Wearable, Low-Cost, Through-Wall Doppler Radar System," International Journal of Antennas and Propagation, vol. 2012.

[4] J. Lilja, V. Pynttari, T. Kaija, R. Makinen, E. Halonen, H Sillanpaa, J. Heikkinen, M. Mantysalo, P. Salonen and P. de Maagt, "Body-Worn Antennas Making a Splash: Lifejacket-Integrated Antennas for Global Search and Rescue Satellite System," Antennas and Propagation 
Magazine, IEEE, vol. 55, no. 2, pp. 324-341, 2013.

[5] A. Pantelopoulos and N. Bourbakis, "A Survey on Wearable Sensor-Based Systems for Health Monitoring and Prognosis," Systems, Man, and Cybernetics, Part C: Applications and Reviews, IEEE Transactions on, vol. 40, no. 1, pp. 1-12, 2010.

[6] I. Locher, M. Klemm, T. Kirstein and G. Troster, "Design and Characterization of Purely Textile Patch Antennas," Advanced Packaging, IEEE Transactions on, vol. 29, no. 4, pp. 777-788, 2006.

[7] A. Tronquo, H. Rogier, C. Hertleer and L. V. Langenhove, "Robust planar textile antenna for wireless body LANs operating in $2.45 \mathrm{GHz}$ ISM band," Electronics Letters, vol. 42, no. 3, pp. 142-143, 2006.

[8] L. Vallozzi, P. V. Torre, C. Hertleer, H. Rogier, M. Moeneclaey and J. Verhaevert, "Wireless Communication for Firefighters Using Dual-Polarized Textile Antennas Integrated in Their Garment," Antennas and Propagation, IEEE Transactions on, vol. 58, no. 4, pp. 1357-1368, 2010.

[9] P. Salonen, Y. Rahmat-Samii, H. Hurme and M. Kivikoski, "Dual-band wearable textile antenna," in Antennas and Propagation Society International Symposium, 2004. IEEE, 2004.

[10] S. Z. Zhu and R. Langley, "Dual-Band Wearable Textile Antenna on an EBG Substrate," Antennas and Propagation, IEEE Transactions on, vol. 57, no. 4, pp. 926-935, 2009.

[11] P. Soh, G. A. E, S. Ooi and M. R. N, "Wearable dualband Sierpinski fractal PIFA using conductive fabric," Electronics Letters, vol. 47, no. 6, pp. 365-367, 2011.

[12] D. Paul, H. Giddens, M. Paterson, G. Hilton and J. McGeehan, "Impact of Body and Clothing on a Wearable Textile Dual Band Antenna at Digital Television and Wireless Communications Bands," Antennas and Propagation, IEEE Transactions on, vol. 61, no. 4, pp. 2188-2194, 2013.

[13] N. M. Rais, P. Soh, M. Malek and G. Vandenbosch, "Dual-Band Suspended-Plate Wearable Textile Antenna," Antennas and Wireless Propagation Letters, IEEE, vol. 12, pp. 583-586, 2013.

[14] M. Klemm and G. Troester, "Textile UWB antennas for wireless body area networks," Antennas and Propagation, IEEE Transactions on, vol. 54, no. 11, pp. 3192-3197, 2006.

[15] P. Salonen, F. Yang, Y. Rahmat-Samii and M. Kivikoski, "WEBGA - wearable electromagnetic band-gap antenna," in Antennas and Propagation Society International Symposium, 2004. IEEE, 2004.

[16] K. Lui, O. Murphy and C. Toumazou, "A Wearable Wideband Circularly Polarized Textile Antenna for Effective Power Transmission on a Wirelessly-Powered Sensor Platform," Antennas and Propagation, IEEE Transactions on, vol. 61, no. 7, pp. 3873-3876, 2013.

[17] M. Jalil, M. Rahim, N. Samsuri, N. Murad, N. Othman and $\mathrm{H}$. Majid, "On-body investigation of dual band diamond textile antenna for wearable applications at 2.45 $\mathrm{GHz}$ and $5.8 \mathrm{GHz}$," in 7th European Conference on Antennas and Propagation (EuCAP), 2013.

[18] R. Moro, S. Agneessens, H. Rogier and M. Bozzi, "Wearable textile antenna in substrate integrated waveguide technology," Electronics Letters, vol. 48, no. 16, pp. 985-986, 2012.

[19] G. Q. Luo, Z. F. Hu, L. X. Dong and L. L. Sun, "Planar Slot Antenna Backed by Substrate Integrated Waveguide Cavity," Antennas and Wireless Propagation Letters, IEEE, vol. 7, pp. 236-239, 2008.

[20] G. Q. Luo, Z. F. Hu, W. J. Li, X. H. Zhang, L. L. Sun and J. F. Zheng, "Bandwidth-Enhanced Low-Profile CavityBacked Slot Antenna by Using Hybrid SIW Cavity Modes," Antennas and Propagation, IEEE Transactions on, vol. 60, no. 4, pp. 1698-1704, 2012.

[21] J. Xu, W. Hong, H. Tang, Z. Kuai and K. Wu, "HalfMode Substrate Integrated Waveguide (HMSIW) LeakyWave Antenna for Millimeter-Wave Applications," Antennas and Wireless Propagation Letters, IEEE, vol. 7 , pp. 85-88, 2008.

[22] S. a. N. M. Razavi, "Development of a Linearly Polarized Cavity-Backed Antenna Using HMSIW Technique," Antennas and Wireless Propagation Letters, IEEE, vol. 11, pp. 1307-1310, 2012.

[23] C. Jin, R. Li, A. Alphones and X. Bao, "Quarter-Mode Substrate Integrated Waveguide and Its Application to Antennas Design," Antennas and Propagation, IEEE Transactions on, vol. PP, no. 99, pp. 1-1, 2013.

[24] T. Kaufmann and C. Fumeaux, "Wearable Textile HalfMode Substrate-Integrated Cavity Antenna using Embroidered Vias," Antennas and Wireless Propagation Letters, IEEE, vol. 12, pp. 805-808, 2013.

[25] P. J. Soh, S. J. Boyes, G. A. Vandenbosch, Y. Huang and S. L. Ooi, "On-body characterization of dual-band alltextile PIFA," Progress In Electromagnetics Research, vol. 129, pp. 517-539, 2012.

[26] "IEEE Standard for Safety Levels With Respect to Human Exposure to Radio Frequency Electromagnetic Fields, $3 \mathrm{kHz}$ to $300 \mathrm{GHz}$," IEEE Std C95.1, 1999 Edition, 1999.

[27] F. Declercq, H. Rogier and C. Hertleer, "Permittivity and Loss Tangent Characterization for Garment Antennas Based on a New Matrix-Pencil Two-Line Method," Antennas and Propagation, IEEE Transactions on, vol. 56, no. 8, pp. 2548-2554, 2008.

[28] "Computer Simulation Technology (CST)," [Online]. Available: http://www.cst.com.

[29] "Agilent Technologies," [Online]. Available: www.agilent.com.

[30] M. L. Scarpello, L. Vallozzi, H. Rogier and D. Vande Ginste, "High-Gain Textile Antenna Array System for Off-Body Communication," International Journal of Antennas and Propagation, vol. 2012. 


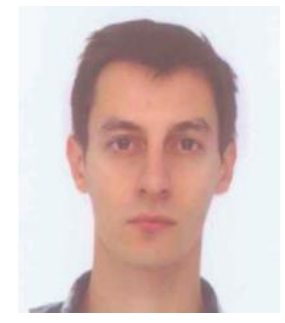

Sam Agneessens was born in Belgium in 1986. He received the B.S. and M.S. degrees in electrical engineering from the University of Ghent, Ghent, Belgium, in 2011.He is currently working towards his Ph.D. within the electromagnetic group in the Department of Information Technology (INTEC) at Ghent University. His research is focuses on the design and development of textile antennas for wearable applications and implementation of Substrate Integrated Waveguide (SIW) techniques on textile materials.

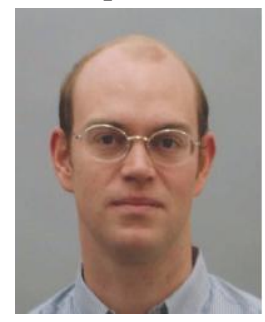

Hendrik Rogier was born in 1971. He received the Electrical Engineering and the Ph.D. degrees from Ghent University, Gent, Belgium, in 1994 and in 1999, respectively.

$\mathrm{He}$ is currently a Full Professor with the Department of Information Technology of
Ghent University, Belgium, Guest Professor at IMEC, Heverlee, Belgium, and Visiting Professor at the University of Buckingham, UK. From October 2003 to April 2004, he was a Visiting Scientist at the Mobile Communications Group of Vienna University of Technology.

He authored and coauthored about 90 papers in international journals and about 110 contributions in conference proceedings. He is serving as a member of the Editorial Board of IET Science, Measurement Technology and acts as the URSI Commission B representative for Belgium. Within the IEEE Microwave Theory and Techniques Society, he is a member of Technical Committee 24 on RFID technology and within the European Microwave Association, he is a member of the Governing Board of Topical Group MAGEO on Microwaves in Agriculture, Environment and Earth Observation. His current research interests are antenna systems, radiowave propagation, body-centric communication, numerical electromagnetics, electromagnetic compatibility and power/signal integrity. 